\title{
Emission channeling studies of Pr in GaN
}

\author{
U. Wahl, A. Vantomme, and G. Langouche \\ Instituut voor Kern- en Stralingsfysica, University of Leuven, Celestijnenlaan 200 D, B-3001 Leuven, Belgium
}

J.P. Araújo

Instituto de Física dos Materiais da Universidade do Porto, University of Porto, P-4150 Porto, Portugal

L. Peralta

Laboratório de Instrumentaçao e Física Experimental de Partículas,

Faculdade de Ciências da Universidade de Lisboa, Av. Elias Garcia 14-1, P-1000-149 Lisbon, Portugal

J.G. Correia and the ISOLDE collaboration

CERN-EP, CH-1211 Geneva 23, Switzerland

(Received 23 November 1999; accepted for publication 26 April 2000)

We report on the lattice location of $\mathrm{Pr}$ in thin film, single-crystalline hexagonal GaN using the emission channeling technique. The angular distribution of $\beta^{-}$particles emitted by the radioactive isotope ${ }^{143} \mathrm{Pr}$ was monitored by a position-sensitive electron detector following $60 \mathrm{keV}$ room temperature implantation of the precursor isotope ${ }^{143} \mathrm{Cs}$ at a dose of $1 \times 10^{13} \mathrm{~cm}^{-2}$ and annealing up to $900^{\circ} \mathrm{C}$. Our experiments provide direct evidence that $\mathrm{Pr}$ is thermally stable at substitutional Ga sites.

\section{Introduction}

Rare earth doped $\mathrm{GaN}$ is a very promising candidate for optoelectronic devices operating in the visible or infrared region [1]. While interest in the past focused mostly on infrared applications, especially Er-doped GaN devices emitting at $1.54 \mu \mathrm{m}$, it has been shown recently that electroluminescence in the red, green and blue can be produced by doping GaN with Eu [2], Er [3], and Tm [4], respectively. Photo- and electroluminescence in the red region of the spectrum $(650 \mathrm{~nm})$ was also obtained from praseodymium (Pr) doped GaN [5-7]. Cathodoluminescence in the visible region of the spectrum has been observed from GaN doped with Dy, Er, Tm [8] and $\mathrm{Sm}$ or Ho [9]. Rare earth doped samples were produced both by doping during growth and by ion implantation at doses around $10^{13}-10^{15} \mathrm{~cm}^{-2}$.

Since the crystal field influences the intensity and splitting of optical transitions, knowledge of the lattice sites and atomic surroundings of the rare earth atoms is required for a detailed understanding of their luminescence properties. To date, a limited number of experiments have explored the structural properties of rare earth elements in $\mathrm{GaN}$. The first lattice location study of rare earth atoms in GaN was performed by Dalmer et al [10] applying the emission channeling technique [11]. This experimental method makes use of the fact that charged particles emitted from radioactive isotopes in a single crystal experience channeling or blocking effects along crystallographic axes and planes. This leads to an anisotropic emission yield from the crystal surface, which depends characteristically on the occupied lattice sites of the probe atoms. In the study of Dalmer et al, the radioactive isotopes ${ }^{167} \mathrm{Tm}\left(t_{1 / 2}=\right.$ $9.25 \mathrm{~d})$ and ${ }^{169} \mathrm{Yb}\left(t_{1 / 2}=32.0 \mathrm{~d}\right)$ were implanted with 60 $\mathrm{keV}$ at doses of $1 \times 10^{13} \mathrm{~cm}^{-2}$. These probe nuclei decay via electron capture (EC) to the excited states of ${ }^{167 \mathrm{~m}} \operatorname{Er}(2.27 \mathrm{~s})$ and ${ }^{169 *} \mathrm{Tm}(0.66 \mu \mathrm{s})$ respectively, which decay to the ground states of the ${ }^{167} \mathrm{Er}$ and ${ }^{169} \mathrm{Tm}$ nuclei. These transitions to the ground states are accompanied by the emission of conversion electrons, which can be used to determine the lattice location of the daughter nuclei ${ }^{167 \mathrm{~m}} \mathrm{Er}$ and ${ }^{169 *} \mathrm{Tm}$. It was found that around $90 \%$ of ${ }^{167 \mathrm{~m}} \mathrm{Er}$ and ${ }^{169 *} \mathrm{Tm}$ atoms were located along the GaN c-axis with a root mean square (rms) displacement of $0.25 \AA$. The channeling patterns were not affected by annealing the implanted samples at $800^{\circ} \mathrm{C}$. The main reason that the sublattice preference could not be derived from the experimental data is the fact that simulations were only available for electron channeling along the c-axis, see also Ref. [12].

The second experiment on the lattice location of rare earth atoms in GaN was done by Alves et al [13, 14], who implanted $\mathrm{Er}$ at $160 \mathrm{keV}$ with doses of $6 \times 10^{14} \mathrm{~cm}^{-2}$ to $6 \times 10^{15} \mathrm{~cm}^{-2}$ and used the Rutherford backscattering/channeling (RBS/C) method. The comparison of angular scans of backscattered ${ }^{4} \mathrm{He}$ around the [0001] and [1011] directions with Monte Carlo channeling simulations revealed that $60-70 \%$ of the $\mathrm{Er}$ atoms were incorporated into substitutional Ga sites for the $6 \times 10^{14} \mathrm{~cm}^{-2}$ implantation. Possible displacements of $\mathrm{Er}$ atoms from the ideal $\mathrm{Ga}$ sites were not considered in the analysis of this experiment. Annealing at $900^{\circ} \mathrm{C}$ caused a decrease of the substitutional $\mathrm{Er}$ fraction except for a sample which was co-implanted with $O$. The $6 \times 10^{15} \mathrm{~cm}^{-2}$ implantation resulted in amorphization of the implanted region, which could not be recovered even by $900^{\circ} \mathrm{C}$ annealing.

Recently, also electron spin resonance experiments have been done on GaN bulk single crystals doped with Er during high-pressure synthesis [15]. The average g-factor 
of $\mathrm{Er}$ was found to be in accordance with theoretical predictions for $\mathrm{Er}^{3+}$ ions on substitutional Ga sites.

In this paper we present a detailed lattice location study of ${ }^{143} \operatorname{Pr}\left(t_{1 / 2}=13.58 \mathrm{~d}\right)$ in $\mathrm{GaN}$ using the emission channeling technique. We have measured electron emission patterns around four different crystallographic directions as a function of annealing temperature, and compared all experimental patterns with simulated yields for a variety of lattice positions. Corrections due to backscattered electrons were included in the quantitative analysis by means of Monte Carlo simulations of electron scattering, taking into account the main geometrical features of our experimental setup. Due to the combination of these experimental and theoretical approaches we are able to quantitatively identify $\mathrm{Pr}$ on Ga sites, to provide values for the average displacement from ideal substitutional sites, and to exclude major fractions on other highly symmetric sites.

\section{Method}

The sample was a commercially available thin film $(1.6 \mu \mathrm{m})$ of not intentionally doped, single-crystalline hexagonal GaN, epitaxially grown on c-axis oriented sapphire by metal-organic chemical vapor deposition (MOCVD). In order to dope with ${ }^{143} \mathrm{Pr}$, we implanted the short-lived isotope ${ }^{143} \mathrm{Cs}(1.78 \mathrm{~s})$, which was produced at CERN's ISOLDE facility [16]. ISOLDE provides ${ }^{143} \mathrm{Cs}$ beams with an approximate intensity of $2 \times 10^{9}$ atoms s ${ }^{-1}$ and $60 \mathrm{keV}$ energy. We implanted a dose of $1 \times 10^{13} \mathrm{~cm}^{-2}$ at an angle of $7^{\circ}$ from the surface direction using a $1 \mathrm{~mm}$ diameter beam spot. ${ }^{143} \mathrm{Cs}$ decays into ${ }^{143} \mathrm{Pr}$ via the decay chain ${ }^{143} \mathrm{Cs}(1.78 \mathrm{~s}) \rightarrow{ }^{143} \mathrm{Ba}(13.5 \mathrm{~s}) \rightarrow{ }^{143} \mathrm{La}(14.0 \mathrm{~min})$ $\rightarrow{ }^{143} \mathrm{Ce} \quad(33.0 \quad \mathrm{~h}) \rightarrow{ }^{143} \mathrm{Pr} \quad(13.58$ d). Following implantation, the sample was therefore stored for two weeks until $99.9 \%$ of ${ }^{143} \mathrm{Ce}$ had transformed into ${ }^{143} \mathrm{Pr}$. Possible influences of this transmutation doping procedure on the lattice sites of ${ }^{143} \mathrm{Pr}$ will be discussed below.

${ }^{143} \mathrm{Pr}$ decays into stable ${ }^{143} \mathrm{Nd}$ by the emission of $\beta^{-}$ particles with an endpoint energy of $935 \mathrm{keV}$. These $\beta^{-}$ particles were recorded by the position-sensitive detection system described in Refs. [17, 18]. Emission channeling patterns around the [0001], [1102], [1101] and [2113] directions of the sample were measured at room temperature in the as-implanted state and after $10 \mathrm{~min}$ isochronal vacuum $\left(<10^{-6}\right.$ mbar $)$ annealing at 200, 400, 600,800 and $900^{\circ} \mathrm{C}$.

A common problem in $\beta^{-}$emission channeling experiments is the effect of electrons that are backscattered from the sample, the sample holder or the walls of the vacuum chamber [11]. A considerable fraction of these electrons reaches the detector, resulting in a rather homogeneous background count rate. Most backscattered electrons have lower energies than the electrons contributing to the channeling effect. Hence it is possible to discriminate this background to a large extent in experiments using conversion electrons since these are monoenergetic particles. The continuous nature of $\beta^{-}$ energy spectra, however, makes this simple correction procedure impossible. As a new approach we have chosen to quantify the backscattering contribution by means of Monte Carlo electron scattering simulations using the EGS4 code [19]. The simulations took into account the relevant elemental composition and main geometrical features of the $\mathrm{GaN}$ thin film, the $\mathrm{Al}_{2} \mathrm{O}_{3}$ substrate, the Mo sample holder and the stainless steel vacuum chamber, but were only run for the geometry where the surface normal of the sample points towards the detector. Simulations for offsurface orientations will also be possible after suitable modifications of the code, taking into account the reduced symmetry. The energy of the emitted electrons was chosen with respect to the energy spectrum of ${ }^{143} \operatorname{Pr}[20]$ while the threshold for detection was $50 \mathrm{keV}$.

According to the simulations, the count rate in the detector is increased by a factor of 1.57 with respect to the number of electrons that were initially emitted within its $0.010 \pi$ solid angle. From this $57 \%$ increase $35 \%$ are due to electrons that were initially emitted within the $2 \pi$ solid angle opposite to the detector and have subsequently been backscattered from the sample or the sample holder. The remaining $22 \%$ increase comes from electrons that were emitted in the $2 \pi$ solid angle towards the sample surface and have reached the detector after scattering at the walls of the chamber. The $35 \%$ increase caused by sample and sample holder may be compared to the value derived from empirical relations for a semi-infinite substrate of average atomic number $Z$ [21]. Considering only the $\mathrm{Al}_{2} \mathrm{O}_{3}$

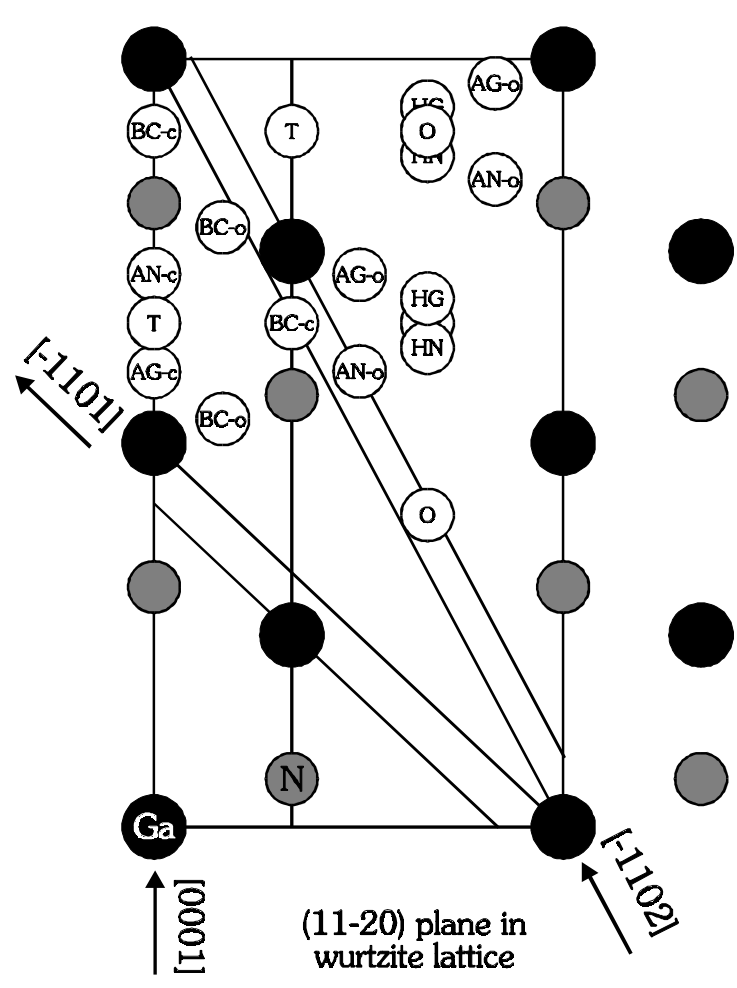

Fig. 1: The (1120) plane in the GaN wurtzite lattice, showing the $\mathrm{Ga}$ and $\mathrm{N}$ lattice positions, and the interstitial sites considered as possible lattice positions of Pr. Note that the O sites are very close to the HG and HN sites, so that the corresponding circles overlap. Along the [1101] and [1102] directions, the double rows of $\mathrm{Ga}$ atoms are indicated (the corresponding $\mathrm{N}$ double rows are not marked). 


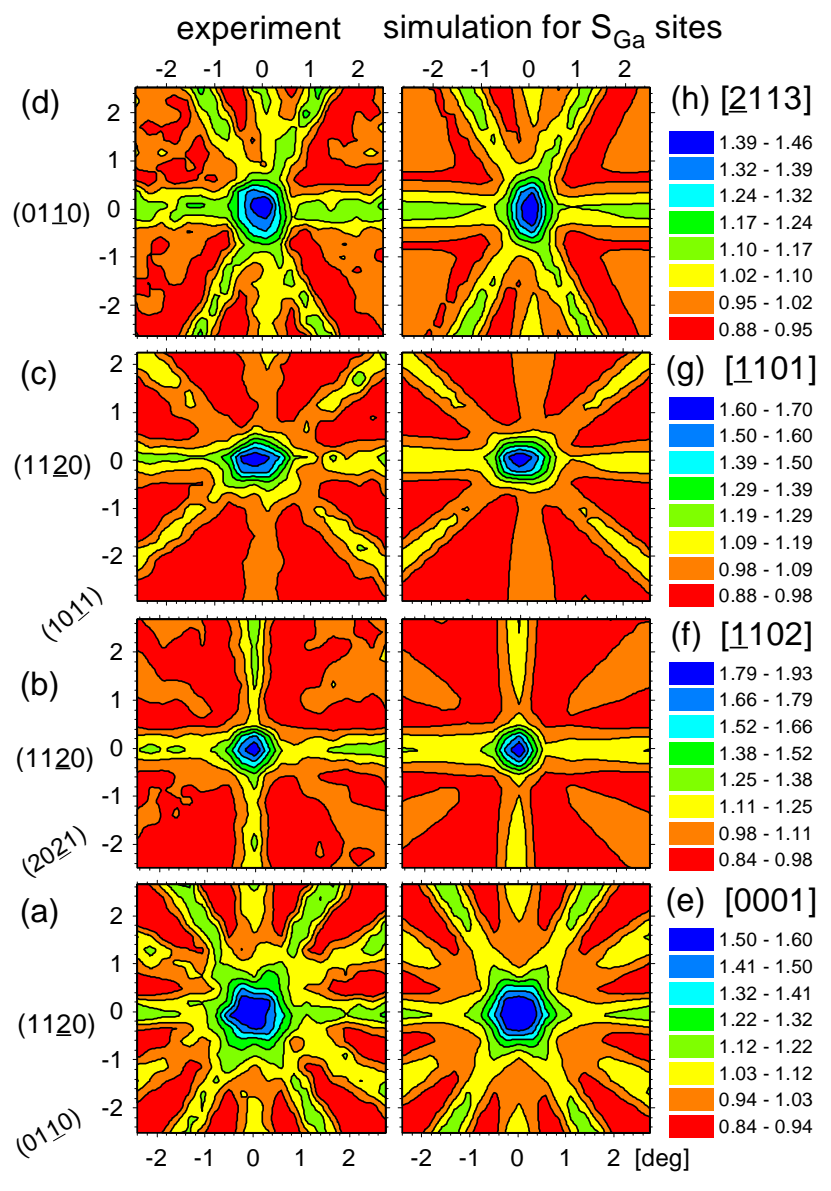

FIG. 2. (a)-(d): Angle-dependent $\beta^{-}$emission yield from ${ }^{143} \mathrm{Pr}$ implanted GaN. (a) and (b) are following annealing at $800^{\circ} \mathrm{C}$, while (c) and (d) are after $900^{\circ} \mathrm{C}$ annealing. (e)-(h): Best fits of simulated channeling patterns for $\mathrm{S}_{\mathrm{Ga}}$ sites to the experimental yields, corresponding to $78 \%, 79 \%, 73 \%$ and $67 \%$ of $\operatorname{Pr}$ on $\mathrm{S}_{\mathrm{Ga}}$ sites.

substrate with $Z=10$, the empirical value is $27 \%$, which is lower than the Monte Carlo result that also takes into account the Mo sample holder. To correct for off-surface orientations, we have assumed that the $22 \%$ increase due to scattering from the walls is independent from the sample orientation, but that the backscattering from the sample and the sample holder scales proportional to the empirical relations, which for $Z=10$ predict increases of $27 \%, 33 \%$ and $41 \%$ under $0^{\circ}, 30^{\circ}$ and $45^{\circ}$ from the surface normal, respectively. In conclusion, a constant background of $36 \%$ was subtracted from the [0001] patterns, $40 \%$ from the [1102] and [2113] and 43\% from the [1101] patterns. We should remark that Compton electrons resulting from the scattering of $\gamma$ rays emitted by the sample (another source of background that is occasionally observed in emission channeling experiments) are absent for ${ }^{143} \mathrm{Pr}$, since this isotope only possesses one $\gamma$ transition with a branching ratio of $1.2 \times 10^{-6}$ [20].

In order to identify the occupied Pr sites, we have compared the experimental channeling data to simulated patterns by means of a two-dimensional fitting procedure as outlined in Ref. [18]. Two-dimensional theoretical patterns for different lattice sites were calculated for

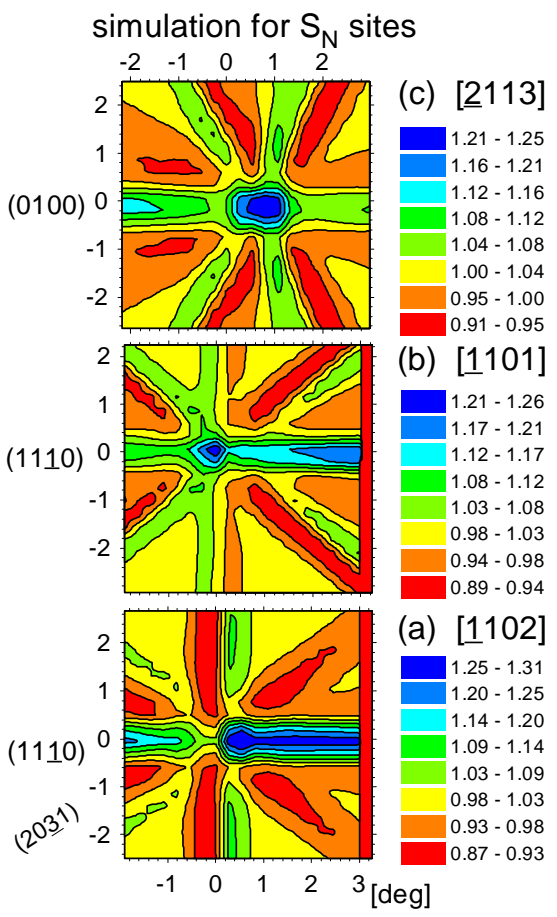

FIG. 3. Best fits of simulated channeling patterns for $S_{N}$ sites to the experimental yields around the (a) [1102], (b) [1101] and (c) [2113] direction, corresponding to $81 \%, 65 \%$ and $40 \%$ of $\operatorname{Pr}$ on $\mathrm{S}_{\mathrm{N}}$ sites, respectively.

emission angles of $0^{\circ}$ to $3^{\circ}$ from the respective crystal directions in steps of $0.05^{\circ}$, using the "many beam" approach for electron channeling in single crystals [11]. The interaction of the electrons with the crystal atoms was modeled by means of Doyle-Turner potentials [22]. In order to describe the GaN wurtzite structure we used lattice constants of $a=3.189 \AA$ and $c=5.185 \AA$ [23] and isotropic room temperature vibration amplitudes of $u_{1}(\mathrm{Ga})=0.074 \AA$ and $u_{1}(\mathrm{~N})=0.081 \AA$. These thermal vibration amplitudes were extracted from X-ray diffraction structure experiments [24] and correspond to Debye temperatures of $\Theta_{D}(\mathrm{Ga})=343 \mathrm{~K}$ and $\Theta_{\mathrm{D}}(\mathrm{N})=739 \mathrm{~K}$. We found that for $\mathrm{GaN}$ "16-beam" simulations, in which the Doyle-Turner potentials are approximated by $33 \times 33$ and the electron wave functions by $17 \times 17$ Fourier components are sufficient to calculate the electron yields to a precision of better than $1 \%$. The electron flux density as a function of depth was weighted by a Gaussian depth distribution of emitter atoms centered at $169 \AA$ with a full width half maximum of $145 \AA$, as was derived from TRIM92 [25] simulations. To approximate the continuous $\beta$ spectrum of ${ }^{143} \mathrm{Pr}$, we calculated theoretical emission yields for electron energies between $50 \mathrm{keV}$ and $925 \mathrm{keV}$ in steps of $25 \mathrm{keV}$. The resulting patterns were averaged, taking into account the $\beta^{-}$energy distribution from the decay of ${ }^{143} \operatorname{Pr}[20]$.

Besides $\mathrm{Pr}$ on substitutional $\mathrm{Ga}$ and $\mathrm{N}$ sites with various vibration amplitudes we also considered the following interstitial sites (Fig. 1): bond-centered sites both within and off the GaN c-axis (abbreviated as BC-c and BC-o), anti-bonding sites AG-c, AN-c, AG-o and AN-o, the "hexagonal" sites $\mathrm{HG}$ and $\mathrm{HN}$, and the so-called T- and 
O-sites [26]. The HG, $\mathrm{HN}$ and $\mathrm{O}$ positions are sites of trigonal $\left(C_{3 v}\right)$ symmetry which are centered in the hexagon spanned by the $\mathrm{Ga}$ and $\mathrm{N}$ c-axis rows, and hence would all produce the same channeling pattern of hexagonal symmetry along the c-axis. Relaxed $\mathrm{T}$ and $\mathrm{O}$ positions have been considered previously as possible sites for native $\mathrm{Ga}$ and $\mathrm{N}$ interstitials [26].

\section{Results}

The [0001] emission channeling pattern of 50-935 keV $\beta^{-}$particles from the implanted $\mathrm{GaN}$ thin film following the annealing sequence to $800^{\circ} \mathrm{C}$ is displayed in Fig. 2(a). The channeling effects along the c-axis and the major crystal planes are evidence that the majority of ${ }^{143} \mathrm{Pr}$ atoms occupy sites which are located within the atomic rows along the c-axis. Note that this does not yet prove the substitutionality of $\mathrm{Pr}$ since there are also interstitial sites located within these rows. The best two-fraction fit of the theoretical yields to the experimental data is shown in Fig. 2(e) and corresponds to $78 \%$ of $\mathrm{Pr}$ atoms with an $\mathrm{rms}$ displacement of $u_{1}(\operatorname{Pr})=0.18 \AA$ from the c-axis, and $22 \%$ on random (R) sites, which are characterized by an isotropic emission yield. Possible reasons for the occurrence of this random fraction are discussed below. Including additional lattice sites off the c-axis in the fit did not result in a significant improvement in the quality of fit.

Suitable directions in order to unambiguously identify the lattice sites of $\operatorname{Pr}$ are the [1102], [1101] and [2113] axes. The [1102] and [1101] directions are indicated in Fig. 1, the [2113] direction is located within the (0110) plane at an angle of $31.6^{\circ}$ from the c-axis. All three directions are characterized by double-rows of $\mathrm{Ga}$ which are well-separated from the corresponding double-rows of $\mathrm{N}$. The emission channeling patterns of these three directions are shown in Figs. 2(b), (c) and (d). Good fits to the experimental data have already been obtained considering only Pr on substitutional Ga sites and on random sites. Figures 2(f), (g) and (h) show the best fits to the [1102], [1101] and [2113] patterns, corresponding to $79 \%, 73 \%$ and $67 \%$ of Pr on $\mathrm{S}_{\mathrm{Ga}}$ with $u_{1}(\mathrm{Pr})=0.14 \AA, 0.10$ $\AA$ and $0.14 \AA$, respectively. The rms displacements from the four directions and their variation suggest a Pr position somewhat displaced from the Ga sites in a specific crystal direction. Fits where $\operatorname{Pr}$ on both $S_{\mathrm{Ga}}$ and $\mathrm{S}_{\mathrm{N}}$ sites were allowed, resulted in fractions on $S_{\mathrm{N}}$ sites of less than $4 \%$ with only a small improvement in the chi square of fit. Allowing for Pr on interstitial sites such as $\mathrm{H}_{\mathrm{Ga}}, \mathrm{H}_{\mathrm{N}}, \mathrm{T}, \mathrm{O}$, etc also did not significantly improve the quality of fit.

The difference in the nuclear charge between gallium $(Z=31)$ and nitrogen $(Z=7)$ is quite large, hence the sublattice preference of the probe atoms has a pronounced influence on the channeling patterns around the [1102], [1101] and [2113] directions. This provides a straightforward sublattice identification, as can be seen by inspecting the fit results considering only $\mathrm{Pr}$ on substitutional $\mathrm{N}$ and random sites [Figs. 3(a)-(c)]. These are clearly not in accordance with the experimental data resulting in fits with chi squares roughly 3 times worse than those shown in Figs. 1(f)-(h). We should also stress

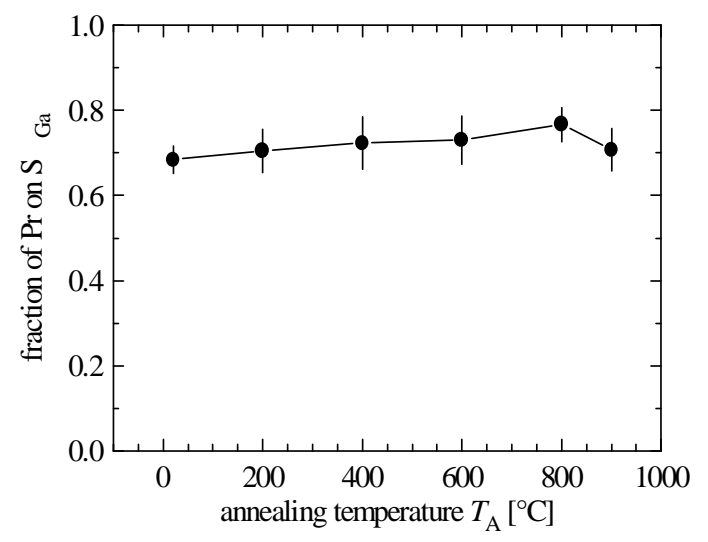

FIG. 4. Fraction of $\operatorname{Pr}$ on substitutional Ga sites as function of isochronal (10 $\mathrm{min})$ annealing temperature.

the lack of mirror symmetry along the (1120) and (0110) planes. While this is characteristic of all [1102], [1101] and [2113] patterns of the wurtzite structure, the simulation results show that it should be much more pronounced for emitters on $\mathrm{N}$ sites than for those on $\mathrm{Ga}$ sites. This is due to the fact that electrons which are emitted from pure Ga rows mainly interact with the relatively broad and deep continuum potential created by the mirror-symmetric double rows of Ga indicated in Fig. 1. The presence of surrounding $\mathrm{N}$ rows, which are responsible for breaking the mirror symmetry, only represents a slight perturbation to the $\mathrm{Ga}$ potential. Correspondingly, the experimental and theoretical patterns in Fig. 1(b)-(d), (f)-(h) are almost mirror-symmetric although some left-right asymmetries are visible. On the other hand, electrons which are emitted from $\mathrm{N}$ rows cannot stay bound in the shallow $\mathrm{N}$ potential and their motion is considerably influenced by the neighboring $\mathrm{Ga}$ rows, hence the pronounced left-right asymmetry of the theoretical patterns in Fig. 3(a)-(c).

Figure 4 shows the average fraction of substitutional $\mathrm{Pr}$ as a function of annealing temperature. In addition to the c-axis pattern at least two off-surface directions were investigated following each annealing step, and the resulting three or four fractions averaged. The error bars in Fig. 4 indicate the corresponding standard deviations, which do not include systematic errors. In direct comparison to the as-implanted state, we only found a small increase in the substitutional Pr fraction following annealing up to $800^{\circ} \mathrm{C}$ which was accompanied by a roughly $0.02 \AA$ decrease in the rms displacement from all four axial directions, reaching the values given above.

\section{Discussion}

We shall first discuss possible influences due to the transmutation doping procedure which we have used in order to incorporate ${ }^{143} \mathrm{Pr}$. The neutrino and beta recoil energies received by the daughter nuclei are roughly around $146 \mathrm{eV}$ for ${ }^{143} \mathrm{Cs} \rightarrow{ }^{143} \mathrm{Ba}, 68 \mathrm{eV}$ for ${ }^{143} \mathrm{Ba} \rightarrow{ }^{143} \mathrm{La}$ and $41 \mathrm{eV}$ for ${ }^{143} \mathrm{La} \rightarrow{ }^{143} \mathrm{Ce}$. While we have not found any values for the displacement energies of $\mathrm{Ga}$ and $\mathrm{N}$ atoms in $\mathrm{GaN}$ in the literature, they should be somewhere in between the values for $\mathrm{Si}(21 \mathrm{eV})$ and diamond $(35 \mathrm{eV})$ 
[28]. Hence at least the first two recoil energies and possibly also the third one should be high enough to reimplant the daughter atoms, so that the initial lattice sites of ${ }^{143} \mathrm{Cs}$ have been masked once the decay chain has reached ${ }^{143} \mathrm{Ce}$. The recoil energies from the ${ }^{143} \mathrm{Ce} \rightarrow{ }^{143} \mathrm{Pr}$ decay, however, are smaller than $7.3 \mathrm{eV}$, so that very likely ${ }^{143} \mathrm{Pr}$ has inherited its lattice site from ${ }^{143} \mathrm{Ce}$. Thus, while the $\beta^{-}$emission channeling method unambiguously measures the position of the ${ }^{143} \operatorname{Pr}$ nucleus with respect to the GaN lattice at the moment of the decay, this position is still influenced to some extent by the previous site of ${ }^{143} \mathrm{Ce}$. On the other hand, the annealing experiments clearly show that once Pr occupies substitutional Ga sites, it is thermally stable up to $900^{\circ} \mathrm{C}$. Since Ce is also a rare earth element with very similar chemical properties to $\mathrm{Pr}$, we conclude that it is most likely that the same results would have been obtained following direct implantation of Pr.

When comparing the present work with previous emission channeling experiments using rare earth elements, we should stress that the use of the ${ }^{167} \mathrm{Tm}$ and ${ }^{169} \mathrm{Yb}$ isotopes is not entirely free from ambiguities either $[10,18,28-30]$. Due to neutrino emission in the EC decay, the two daughter nuclei receive recoil energies of 0.7-0.9 $\mathrm{eV}$ in the case of ${ }^{167 \mathrm{~m}} \mathrm{Er}$ and $0.7-1.1 \mathrm{eV}$ in the case of ${ }^{169 *} \mathrm{Tm}$ (note that the values of $1.8 \mathrm{eV}$ and $2.6 \mathrm{eV}$ given in Ref. [10] are wrong since it has not been taken into account that the decays lead to excited states of the daughter nuclei). While energies in the $\mathrm{eV}$ region are certainly too small to displace the daughter atoms far from the initial lattice sites of ${ }^{167} \mathrm{Tm}$ and ${ }^{169} \mathrm{Yb}$, thermalization of the recoil could lead to metastable configurations in the lattice. Additional complications could occur from the fact that due to the EC decay the initial electronic configurations of both daughter atoms contain a hole in the $\mathrm{K}$ shell. In the subsequent electronic deexcitation via X-ray emission, a process which occurs before the emission of the conversion electrons, a transfer of electronic to configurational excitation could also result in a change of the structural properties. Such effects have for instance been observed by perturbed angular correlation (PAC) spectroscopy of ${ }^{111} \mathrm{In}$ $\mathrm{H}$ pairs in Si [31]. An approach that may overcome these ambiguities, is to measure the emission channeling effects from ${ }^{167 \mathrm{~m}} \mathrm{Er}$ and ${ }^{169 *} \mathrm{Tm}$ in situ at high temperatures, where potential metastable configurations have a greater possibility of relaxing. We have previously demonstrated this for ${ }^{167 \mathrm{~m}} \mathrm{Er}$ at $500^{\circ} \mathrm{C}$ in $\mathrm{GaAs}\left[30\right.$ ] and at $900^{\circ} \mathrm{C}$ in $\mathrm{Si}$ $[28,29]$, where we were able to show that in both cases the same lattice sites were occupied as at room temperature. For GaN such high-temperature measurements with ${ }^{167 \mathrm{~m}} \mathrm{Er}$ and ${ }^{169 *} \mathrm{Tm}$ have not been done. However, the fact that the average displacement from the c-axis, $0.25 \AA$, is similar to the value of $0.18 \AA$ which we derived for ${ }^{143} \mathrm{Pr}$, indicates that the EC decay is probably not a critical issue.

Our observations of $\operatorname{Pr}$ on substitutional $\mathrm{Ga}$ sites supplement the previous studies that have investigated the lattice location of rare earths in $\mathrm{GaN}$ [10, 13-15]. The preference of rare earth atoms for Ga sites has also been observed for ${ }^{167 \mathrm{~m}} \mathrm{Er}$ in the cubic III-V semiconductor GaAs [30], although in this case a smaller amount of Er was found on tetrahedral interstitial $\mathrm{T}_{\mathrm{As}}$ sites. For $\mathrm{Pr}$ in $\mathrm{GaN}$ we have not seen any indication for such a well-defined interstitial fraction. Possible reasons for the derived fraction on random sites are $\operatorname{Pr}$ atoms in disordered surroundings or a mixture of small fractions of $\operatorname{Pr}$ on various low-symmetry lattice sites. In addition, we cannot exclude an underestimation of the background correction, in which case the maximum substitutional fraction would be higher than $78 \%$. All three possibilities would contribute a nearly homogeneously distributed count rate in the detector.

The annealing behavior of implanted $\mathrm{Pr}$ in $\mathrm{GaN}$ is comparable to what has been reported from studies with other elements (for a review see Ref. [23]). Even in the asimplanted state a high fraction of substitutional $\mathrm{Pr}$ is obtained in accordance with the fact that damage accumulation is not very efficient in $\mathrm{GaN}$ and that it is not easily amorphized. On the other hand, the remaining damage in $\mathrm{GaN}$ is difficult to anneal. Incomplete annealing of implantation disorder and the absence of a pronounced recovery step up to $900^{\circ} \mathrm{C}$ has been reported previously [10, 12-14, 23]. In accordance with this we also did not observe a major recovery step up to $900^{\circ} \mathrm{C}$, although the decrease in the rms displacement of Pr indicates some local atomic rearrangement. The small decrease in the channeling effects following vacuum annealing at $900^{\circ} \mathrm{C}$ (Fig. 4) might be due to $\mathrm{N}$ loss, leaving behind a disordered region near the surface. It is known that $\mathrm{GaN}$ decomposes during high temperature annealing if the surface is not capped or exposed to $\mathrm{N}_{2}$ pressure [32].

\section{Conclusions}

Our experiments show that substitutional Ga sites are thermally stable lattice positions for $\operatorname{Pr}$ in GaN. While for annealing temperatures up to $900^{\circ} \mathrm{C}$ Pr was most likely still somewhat off-center from the Ga sites, we did not find evidence for substantial fractions on other, highly symmetric lattice sites such as substitutional $\mathrm{N}$, or interstitial $\mathrm{HG}, \mathrm{HN}, \mathrm{T}$ or $\mathrm{O}$ sites. By means of measuring the electron emission yield in three off-surface directions and comparison with detailed simulation calculations, we have shown for the first time how to unambiguously identify the sublattice preference of emission channeling probe atoms in GaN. We found that the background correction in $\beta^{-}$emission channeling can be substantially improved with the help of Monte Carlo electron scattering simulations. This procedure should also be helpful for lattice location studies of other probe atoms and different host crystals.

\section{Acknowledgements}

This work was partially funded by the TMR-LSF program of the European Union, and by the FCT, Portugal, through project CERN/c/FIS/15180/99.

[1] A.J. Steckl and J.M. Zavada, MRS Bulletin 24/9, 33 (1999).

[2] J. Heikenfeld, M. Garter, D.S. Lee, R. Birkhahn, and A.J. Steckl, Appl. Phys. Lett. 75, 1189 (1999).

[3] A.J. Steckl and R. Birkhahn, Appl. Phys. Lett. 73, 1702 (1998). 
[4] A.J. Steckl, M. Garter, D.S. Lee, J. Heikenfeld, and R. Birkhahn, Appl. Phys. Lett. 75, 2184 (1999).

[5] R. Birkhahn, M. Garter, and A.J. Steckl, Appl. Phys. Lett. 74, 2161 (1999).

[6] L.C. Chao and A.J. Steckl, Appl. Phys. Lett. 74, 2364 (1999).

[7] J.M. Zavada, R.A. Mair, C.J. Ellis, J.Y. Lin, H.X. Jiang, R.G. Wilson, P.A. Grudowski, and R.D. Dupuis, Appl. Phys. Lett. 75, 790 (1999).

[8] H.J. Lozykowski, W.M. Jadwisienczak and I. Brown, Sol. State Comm. 110, 253 (1999).

[9] H.J. Lozykowski, W.M. Jadwisienczak and I. Brown, Appl. Phys. Lett. 74, 1129 (1999).

[10] M. Dalmer, M. Restle, A. Stötzler, U. Vetter, H. Hofsäss, M.D. Bremser, C. Ronning, R.F. Davis, and the ISOLDE collaboration, Mat. Res. Soc. Proc. 482, 1021 (1998).

[11] H. Hofsäss and G. Lindner, Phys. Rep. 210, 121 (1991).

[12] C. Ronning, M. Dalmer, M. Uhrmacher, M. Restle, U. Vetter, L. Ziegler, H. Hofsäss, T. Gehrke, K. Järrendahl, R.F. Davis, and the ISOLDE collaboration, J. Appl. Phys. 87, 2149 (2000).

[13] E. Alves, M.F. da Silva, J.C. Soares, R. Vianden, J. Bartels, and A. Kozanecki, Nucl. Instr. Meth. B 147, 3383 (1999).

[14] E. Alves, M.F. da Silva, J.C. Soares, J. Bartels, R. Vianden, C.R. Abernathy, and S.J. Pearton, MRS Internet J. Nitride Semicond. Res. 4S1, G11.2 (1999).

[15] M. Palczewska, A. Wolos, M. Kaminska, I. Grzegory, M. Bockowski, S. Krukowski, T. Suski, and S. Porowski, Sol. State Comm. 114, 39 (2000).

[16] E. Kugler, D. Fiander, B. Jonson, H. Haas, A. Przewloka, H.L. Ravn, D.J. Simon, K. Zimmer, and the ISOLDE collaboration, Nucl. Instr. Meth. B 70, 41 (1992).

[17] P. Weilhammer, E. Nygård, W. Dulinski, A. Czermak, F. Djama, S. Gadomski, S. Roe, A. Rudge, F. Schopper and J. Strobel, Nucl. Instr. Meth. A 383, 89 (1996).

[18] U. Wahl, J.G. Correia, S. Cardoso, J.G. Marques, A. Vantomme, G. Langouche and the ISOLDE collaboration, Nucl. Instr. Meth. B 136, 744 (1998).

[19] W.R. Nelson, H. Hirayama, D.W.O. Rogers, The EGS4 Code System, SLAC-Report-265, December 1985; http://www.slac.stanford.edu/pubs/slacreports/slac-r-265.html

[20] S.Y.F. Chu, L.P. Ekström and R.B. Firestone, WWW Table of Radioactive Isotopes, database version of 28.2.1999, http://nucleardata.nuclear.lu.se/toi/.

[21] H.H. Seliger, Phys. Rev. 88, 408 (1952).

[22] P.A. Doyle and P.S. Turner, Acta Cryst. A 24, 390 (1968).

[23] S.J. Pearton, J.C. Zolper, R.J. Shul, and F. Ren, J. Appl. Phys. 86, 1 (1999).

[24] A. Yoshiasa, K. Koto, H. Maeda and T. Ishii, Jpn. J. Appl. Phys. 36, 781 (1997).

[25] J.F. Ziegler, J.P. Biersack and U. Littmark, The Stopping and Range of Ions in Solids (Pergamon Press, New York, 1985).

[26] P. Boguslawski, E.L. Briggs and J. Bernholc, Phys. Rev B 51, 17255 (1995).

[27] J. Bourgoin and M. Lannoo, Point Defects in Semiconductors II: Experimental Aspects, Springer, Berlin, 1983.

[28] U. Wahl, A. Vantomme, J. De Wachter, R. Moons, G. Langouche, J.G. Marques, J.G. Correia, and the ISOLDE collaboration, Phys. Rev. Lett. 79, 2069 (1997).

[29] U. Wahl, J.G. Correia, G. Langouche, J.G. Marques, A. Vantomme, and the ISOLDE collaboration, Mater. Sci. Forum 258-263, 1503 (1997).

[30] U. Wahl, A. Vantomme, G. Langouche, and the ISOLDE collaboration, Nucl. Instr. Meth. B 148, 492 (1999).
[31] H. Skudlik, M. Deicher, R. Keller, R. Magerle, W. Pfeiffer, P. Pross, E. Recknagel, and T. Wichert, Phys. Rev. B 46, 2159 (1992).

[32] R. Groh, G. Gerey, L. Bartha, and J.I. Pankove, Phys. Status Solidi A26, 353 (1974). 